\title{
Lymphomagenic properties of a HIV p17 variant derived from a splenic marginal zone lymphoma occurred in a HIV-infected patient.
}

Francesca Caccuri ${ }^{1}$, Elena Muraro ${ }^{2}$, Annunziata Gloghini ${ }^{3}$, Ombretta Turriziani ${ }^{4}$, Mara Riminucci ${ }^{4}$, Cinzia Giagulli ${ }^{1}$, Katy Mastorci ${ }^{2}$, Damiana Antonia Fae ${ }^{2}$, Simona Fiorentini ${ }^{1}$, Antonino Carbone ${ }^{5}$, Arnaldo Caruso ${ }^{1 \#}$, Riccardo Dolcetti ${ }^{2,6^{* \#}}$.

${ }^{1}$ Section of Microbiology, Department of Molecular and Translational Medicine, University of Brescia, Brescia, Italy. ${ }^{2}$ Immunopathology and Biomarker Unit, Department of Translational Research, Centro di Riferimento Oncologico di Aviano (CRO), IRCCS, Italy. ${ }^{3}$ Department of Pathology and Laboratory Medicine, Fondazione IRCCS, Istituto Nazionale dei Tumori, Milano, Italy. ${ }^{4}$ Department of Molecular Medicine, Sapienza University of Rome, Rome, Italy. ${ }^{5}$ Department of Pathology, Centro di Riferimento Oncologico di Aviano (CRO), IRCCS, Italy. ${ }^{6}$ University of Queensland Diamantina Institute, Translational Research Institute, Brisbane, Australia.

\# Shared senior authorship

Running title: HIV p17 variant in splenic marginal zone lymphoma

Keywords: marginal zone lymphoma, HIV, p17, B lymphocytes, lymphomagenesis

Funding information: Associazione Italiana per la Ricerca sul Cancro; Grant numbers: 14287 (to R.D.) and 20108 (to A.Caru.)

Word count: 3093

This is the author manuscript accepted for publication and has undergone full peer review but has not been through the copyediting, typesetting, pagination and proofreading process, which may lead to differences between this version and the Version of Record. Please cite this article as doi: 10.1002/hon.2562

This article is protected by copyright. All rights reserved. 
* Correspondence: Riccardo Dolcetti, University of Queensland Diamantina Institute, Translational Research Institute, 37 Kent St. Woolloongabba, 4102, Australia. E-mail: r.dolcetti@uq.edu.au Phone: +61 (0)7 34436953. 


\begin{abstract}
Despite antiretroviral therapy, $\mathrm{HIV}^{+}$individuals still have increased risk to develop lymphomas, including marginal zone lymphomas, suggesting that factors other than HIV-related immunosuppression are probably acting as lymphomagenic factors in the HIV setting. The possible pathogenic involvement of HIV p17 protein variants was investigated in a particularly informative case of HIV-related splenic marginal zone lymphoma, who was negative for oncogenic virus infections, thus allowing us to assess the possible direct contribution of these HIV-encoded proteins to lymphomagenesis. The presence of p17 protein was analyzed by immunohistochemistry in lymphoma tissue. Recombinant p17 protein derived from the dominant sequence detected in plasma and lymphoma biopsy was characterized for B-cell proliferation, clonogenicity in soft agar, in vitro tube formation and wound-healing. Intracellular signaling was investigated by immunoblotting. HIV p17 protein was detected in reactive lymphoid follicles but not within lymphoma cells. An identical dominant variant p17 sequence, p17-Lyrm, carrying a 117-118 Ala-Ala insertion was detected in both plasma and lymphoma tissue. Recombinant p17-Lyrm enhanced B-cell proliferation and clonogenicity, promoted the formation of capillary-like structures and enhanced endothelial cell migration. Unlike reference p17, the p17-Lyrm variant enhanced the activation of Akt and ERK, critical kinases in lymphomagenesis. p17-Lyrm clonogenic activity was dependent on the activation of Akt but not of ERK1/2. These results indicate the HIV p17 variants with distinct molecular signatures and functional properties may accumulate in lymphoid tissues of HIVinfected individuals where they may act as a local stimulus promoting the development of lymphomas.
\end{abstract}




\section{Introduction}

The widespread use of combination antiretroviral therapy (cART) has significantly decreased the risk of lymphoma in Human Immunodeficiency Virus (HIV)-infected population. ${ }^{\mathbf{1} 2}$ However, despite induction of virologic suppression and immune recovery by cART, lymphoma incidence remains much higher than in the general population., ${ }^{\mathbf{3 , 4}}$ These findings suggest that factors other than HIV-related immunosuppression are probably still acting as lymphomagenic contributors in this setting. Although HIV infection markedly increases the risk of AIDS-defining non-Hodgkin lymphoma (NHL) subtypes, such as diffuse large B-cell lymphomas and Burkitt lymphomas, a large population-based study disclosed significantly elevated risks also for other NHL subtypes, particularly marginal zone lymphomas. ${ }^{4}$ These results are consistent with a pathogenic role of HIV also in the development of these relatively uncommon lymphomas in HIV-infected individuals. Notably, the estimated risk for marginal zone lymphomas is similar in patients with HIV infection

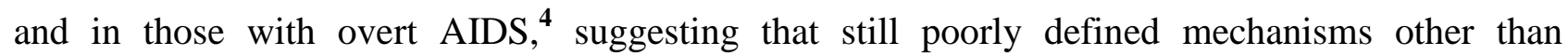
immunodeficiency are probably involved in the pathogenesis of this lymphoma subtype.

Despite the inability of HIV to infect B lymphocytes, recent evidence suggests that HIV may directly contribute to B-cell lymphomagenesis through the pleiotropic effects of viral proteins released in the microenvironment by infected cells, including gp120, Tat, and p17. ${ }^{\mathbf{5}}$ In particular, 
recent evidence indicates that p17 expression in mice transgenic for a defective HIV-1 provirus is associated with lymphoma development. ${ }^{6,7}$ Moreover, unlike the prototype HIV-clade B p17 and p17 proteins derived from HIV-positive individuals without lymphoma, p17 variants derived from a Ugandan HIV-1 A1 strain or from HIV-related NHL are able to activate the PI3K/Akt signaling and enhance B-cell proliferation and clonogenicity. ${ }^{\mathbf{8 9}}$ The p17 matrix protein is continuously released in the extracellular space from HIV-infected cells, ${ }^{\mathbf{1 0}}$ and can be detected in plasma and tissues of HIVinfected patients. ${ }^{11-13}$ Notably, in lymphoid tissues, p17 accumulates and persists even during cART and in the absence of any HIV replicative activity, ${ }^{\mathbf{1 3}}$ thus suggesting that its persistence in the tissue microenvironment, even during pharmacological therapy, may promote chronic B-cell stimulation.

Here we report the characterization of a rare case of splenic marginal zone lymphoma (SMZL) occurred in a HIV-1-infected patient for the presence in the plasma and pathologic tissues of a HIV p17 variant (p17-Lyrm) endowed with functional properties that could have contributed to the development of this lymphoma in the absence of oncogenic virus infections. 


\section{Materials and Methods}

Patient characteristics. The main clinico-pathologic features of the SMZL occurred in a 50-yearold woman with HIV-1 infection were previously described. ${ }^{\mathbf{1 4}}$ In the present study, approved by local IRBs, samples of splenic tumor biopsy and plasma of the patient were used.

Immunohistochemistry. HIV p17 protein expression was evaluated on $3 \mu \mathrm{m}$ formalin-fixed paraffin-embedded tissue sections with the MBS-3 antibody (Ab) ${ }^{\mathbf{1 2}}$ and carried out on a BenchMark Ultra Platform using the OptiviewDAB Detection Kit (Ventana, Tucson, AZ).

PCR and sequencing. Because at the time of sampling the SMZL patient showed a low-level HIV viremia, we used a nested PCR approach amplifying a 559-nucleotide (nt)-long amplicon (nt 326$885)$ to evaluate the whole p17 coding sequence. ${ }^{9}$ Amplification of the same target DNA was repeated at least 5 times.

Recombinant p17 proteins. The coding sequence of HIV-1 isolate BH10 p17 (refp17; amino acids 1-132) and NHL-a101 were amplified and cloned as described. ${ }^{\mathbf{9}, 15}$ The most representative coding sequence of p17-Lyrm, obtained from plasma and biopsy of the SMZL patient was cloned into BamHI and ECORI sites of the pGEX-2T expression vector (Pharmacia, Stockholm, Sweden). Recombinant proteins (in their monomeric form) were purified (>98\%) by reverse-phase fast performance liquid chromatography, as described. ${ }^{\mathbf{1 5}}$ Limulus amoebocyte assay was used to determine the absence of endotoxin contamination $(<0.25$ units $/ \mathrm{ml})$.

Cell Cultures. Human lymphoma B-cell lines HBL1 and Raji (ATCC, 2014) were cultured in RPMI1640 containing 10\% (vol/vol) fetal bovine serum (FBS). Human umbilical vein endothelial 
cells (HUVECs) were isolated as described ${ }^{\mathbf{1 6}}$ and cultured in endothelial growth medium (EGM) (Lonza, Milan) containing 10\% FBS. All experiments were performed with cells at passages 2-6. All cell lines were authenticated by fingerprinting (Power Plex 1.2, Promega) before the start of the study. Peripheral blood mononuclear cells (PBMCs) were obtained from healthy donors who provided written informed consent. Primary B lymphocytes were negatively isolated using the Bcell isolation kit II from Miltenyi Biotech (Bergisch Gladbach, Germany) and cultured in RPMI1640, containing $2 \mathrm{mM}$ l-glutamine, 10\% FBS (Gibco, Grand Island, NY), 100 $\mu \mathrm{g} / \mathrm{ml}$ streptomycin and 100IU/ml penicillin (Sigma-Aldrich, St. Louis, MO), at $37{ }^{\circ} \mathrm{C}$ in $5 \%$ of $\mathrm{CO}_{2}$. Cells were activated with $1 \mu \mathrm{g} / \mathrm{ml}$ of soluble CD40 ligand (CD40L; Alexis, Butler Pike, PA), $1 \mu \mathrm{g} / \mathrm{ml}$ of the corresponding enhancer and 10ng/ml of IL-4 (R\&D Systems, Minneapolis, MN) for 7d, or infected with the B95.8 EBV strain for 10d.

Proliferation assays. HBL-1 cell proliferation was assessed after an overnight starvation, culturing cells in $1 \% \mathrm{FBS}$ with or without $1 \mu \mathrm{g} / \mathrm{ml}$ of p17 proteins for $48 \mathrm{~h}$. Ki-67 expression was evaluated in cells fixed with $2 \%$ paraformaldehyde in complete medium for $10 \mathrm{~min}$ at room temperature, washed in complete medium and permeabilized using $90 \%$ methanol diluted in $\mathrm{H}_{2} \mathrm{O}$. After 10 min. incubation in ice, cells were washed in permeabilization buffer (PBS with 0.5\%BSA; SigmaAldrich) and stained with anti-Ki-67 $\mathrm{Ab}$ in $100 \mu \mathrm{l}$ of $2 \%$ rabbit serum diluted in permeabilization buffer at $4^{\circ} \mathrm{C}$ for $30 \mathrm{~min}$. Samples were washed twice and re-suspended in PBS with $1 \%$ paraformaldehyde for flow cytometry analysis.

Primary B-cell proliferation was assessed by carboxyfluorescein diacetate, succinimidyl ester (CFSE) dilution (Molecular Probes, Eugene, OR). Primary B cells activated with CD40L for 7d or 
EBV-immortalized for $10 \mathrm{~d}\left(2 \times 10^{6} / \mathrm{ml}\right)$ were stained in PBS with $0.5 \mu \mathrm{M} \mathrm{CFSE}$ for $15 \mathrm{~min}$. at $37^{\circ} \mathrm{C}$, washed once and kept in complete medium for further $30 \mathrm{~min}$. at $37^{\circ} \mathrm{C}$. Cells were finally washed twice and cultured $\left(10^{6} / \mathrm{ml}\right)$ in complete medium with p17 proteins. Proteins were restored every $3 \mathrm{~d}$. Cytofluorimetric analysis was performed with a Cytomics FC500 and data analyzed with CXP (Beckman Coulter, Fullerton, CA) and FlowJo (Tree Star, Ashland, OR) softwares.

Soft Agar Anchorage-Independent Growth. Raji cells (12,500/well) were plated in 12-well plates in $2 \mathrm{ml} \mathrm{RPMI}$ containing 5\%FBS and $0.35 \%$ and $0.5 \%$ Sea-Plaque agarose (Lonza), respectively, over a $0.7 \%$ agarose base. One day after plating, medium containing treatments was added to the top of the layer and replaced every $4 \mathrm{~d}$. After 10d, $300 \mu \mathrm{l}$ of 3-[4, 5-Dimethylthiazol-2-y1]-2, 5diphenyltetrazolium bromide (MTT) (Sigma) were added and allowed to incubate for $4 \mathrm{~h}$ at $37{ }^{\circ} \mathrm{C}$. Plates were then placed overnight at $4^{\circ} \mathrm{C}$, and colonies $>50 \mu \mathrm{m}$ in diameter were counted.

B-cell colony formation. Raji cell suspension was sequentially diluted, and cells seeded by manual pipetting into a 96-well plate at a dilution of 0.5 cells/well. Plates were incubated for $8 \mathrm{~d}$ under standard conditions in the presence or absence of $0.01 \mu \mathrm{g} / \mathrm{ml}$ p17-Lyrm alone or in combination with the PI3K/Akt inhibitor Akt VIII ( $1 \mu \mathrm{M}$; Sigma) or the mitogen-activated protein kinase/ERK1/2 inhibitor PD98059 (10 $\mu \mathrm{M}$; Calbiochem). Eight $\mathrm{d}$ after, culture plates were analyzed for single colony formation.

Viable cell counting. Propidium Iodide (PI) staining (Immunostep) was used to detect $\mathrm{PI}^{-}$viable cells by flow cytometry. Absolute cell counts were obtained by the counting function of the MACSQuant ${ }^{\circledR}$ Analyzer (Miltenyi).

This article is protected by copyright. All rights reserved. 
In Vitro Tube Formation. HUVECs were nutrient starved for $24 \mathrm{~h}$ in endothelial basal medium with $0.5 \% \mathrm{FBS}$ and then harvested and re-suspended in EGM containing 10\%FBS. Cells were seeded in growth factor-reduced Cultrex basement membrane extract (Trevigen, Gaithersburg, MD)-coated wells $\left(5 \times 10^{4} /\right.$ well) and treated or not with $0.01 \mu \mathrm{g} / \mathrm{ml}$ of refp 17 or p17-Lyrm.

Wound Healing Assay. HUVECs were plated into $24-w e l l$ plates (10 5 cells/well) in EGM containing $10 \%$ FCS. Confluent monolayers were nutrient starved for $24 \mathrm{~h}$ and then scratched using a $200 \mu 1$ pipette tip. After washing, cells were treated or not with refp17 or p17-Lyrm.

Immunoblotting. After $24 \mathrm{~h}$ starving by serum deprivation (RPMI containing $1 \mathrm{mM}$ L-glutamine and $0.5 \%$ FBS $)$, Raji cells $\left(7 \times 10^{5} / \mathrm{ml}\right)$ were treated with different concentrations of p17 proteins $(0.05,0.1$ and $0.5 \mu \mathrm{g} / \mathrm{ml})$. Immunoblotting was carried out as described ${ }^{8,9}$ using monoclonal Abs to pAkt (Ser473), Akt (Cell Signaling), pERK1/2 (Thr202/Tyr204) and a rabbit polyclonal Ab to ERK1/2 (Santa Cruz).

\section{Results}

\section{HIV p17 is present in tumor microenvironment of SMZL.}

Immunohistochemical analysis showed that expression of HIV p17 was restricted to splenic lymphoid follicles surrounded by lymphoma cells and to scattered endothelial cells located within SMZL (Fig. 1). Conversely, no evidence of p17 was detected in neoplastic B lymphocytes. These findings are consistent with previous observations ${ }^{\mathbf{1 2 , 1 7}}$ and confirm the presence of HIV p17 protein in the tumor microenvironment of this lymphoma.

This article is protected by copyright. All rights reserved. 
p17 gene sequencing in plasma and SMZL tissue.

Nested PCR successfully detected p17 DNA in both plasma and lymphoma tissue, consistently with the immunohistochemical detection of HIV p17 in SMZL tissue, clearly indicating that HIV-1infected cell reservoirs were present in the spleen involved by this lymphoma, even with a very low level of circulating virus. Consistently with previous findings, ${ }^{\mathbf{9}}$ an identical dominant variant p17 (vp17) sequence, p17-Lyrm, was detected in both plasma and lymphoma tissue of this patient (Fig 2A). Notably, the sequence of p17-Lyrm showed the same insertion of an Ala-Ala stretch between amino acids 117 and 118 that we previously detected in clonogenic vp17 proteins derived from two HIV-related NHLs (NHL-a101 and NHL-a104) ${ }^{9}$ (Fig 2A).

\section{Recombinant p17-Lyrm promotes lymphoma B-cell colony formation.}

Recombinant p17-Lyrm was obtained from the dominant sequence detected in lymphoma tissues of this patient and investigated for its ability to enhance clonogenic activity of Raji lymphoma cells. As shown in Fig. 2B, p17-Lyrm, at a concentration ranging from 0.01 to $0.1 \mu \mathrm{g} / \mathrm{ml}$, significantly increased the number of Raji cell colonies in soft agar compared with untreated cells. The extent of clonogenic activity of p17-Lyrm was similar to that of the vp17 NHL-a101 used as a positive control, whereas reference p17 (refp17) was unable to increase the colony-forming ability of Raji cells (Fig. 2B).

\section{p17-Lyrm enhances the proliferation of normal, activated and neoplastic B cells.}

We also assessed the ability of p17-Lyrm to promote the growth of primary, EBV-activated and fully neoplastic B lymphocytes. To this end, we first used primary B cells from two unrelated 
donors pre-activated for 7 days with CD40L and cultured for 3 days in the presence of p17-Lyrm, refp17 or the clonogenic S75X p17 variant. This latter protein was used as positive control due to its growth-promoting ability demonstrated by our previous work. ${ }^{\mathbf{8 , 9 , 1 8}}$ As shown in Fig. 3, all three p17 protein variants induced a slight but detectable increase in primary B-cell proliferation, although with a certain degree of variability between the two donors. Similar findings were obtained when the same experiment was carried out with primary B lymphocytes activated by EBV infection for 10 days and analyzed after 4 days of culture with the recombinant p17 proteins (Fig. 4A). We also investigated the effects of p17 variants on Ki-67 expression of the HBL1 cell line derived from a HIV-related NHL. After culture for 48 hours in 1\% FCS, the clonogenic p17-Lyrm and S75X variants increased, whereas refp17 slightly decreased, the percentage of Ki-67 ${ }^{+}$HBL1 cells (Fig. 4B), consistently with previous findings indicating opposite effects of refp17 and clonogenic variants in fully transformed B lymphocytes.

\section{p17-Lyrm promotes capillary-like structures formation and cell migration.}

Considering that HIV p17 may promote angiogenesis, ${ }^{\mathbf{1 2}}$ we investigated the ability of p17-Lyrm to form capillary-like structures. To this end, HUVEC cells were stimulated for 8 hours at $37^{\circ} \mathrm{C}$ with medium alone or with ref-p17 or p17-Lyrm $(0.01 \mu \mathrm{g} / \mathrm{ml})$. As shown in Fig. 5A, both p17 proteins formed a consistent network of capillary-like structures.

To investigate the effect of p17 on endothelial cell migratory activity, the wound healing assay was performed. This method allows the investigation of the ability of the viral protein to modulate cell migration by sealing a confluent cell monolayer after a mechanical injury. Confluent HUVEC monolayers were scratched with a $200 \mu \mathrm{l}$ tip, and the percentage of wound healing was observed 
during a period of 12 hours. As shown in Fig. 5B, untreated HUVECs reached $\approx 35 \%$ healing only after 12 hours of culture, whereas at the same time HUVECs treated with refp17 or p17-Lyrm reached $100 \%$ healing, showing a considerable improvement in scrape wound repair ability.

\section{p17-Lyrm activates Akt and ERK signaling pathways in B cells}

We have previously shown that most of the biologic effects induced by lymphoma-derived p17 proteins were associated with the activation of the Akt and ERK pathways. ${ }^{\mathbf{8 , 9 , 1 8}}$ Considering that activation of these B-cell growth-promoting signalings could have played a role also in the pathogenesis of this SMZL, we investigated the ability of p17-Lyrm to enhance the activating phosphorylation of Akt and ERK. Raji cells stimulated with p17-Lyrm significantly increased the level of the active phosphorylated Akt kinase at all protein concentrations used (Fig. 6A). Moreover, at the concentration of $0.05 \mu \mathrm{g} / \mathrm{ml}, \mathrm{p} 17$-Lyrm significantly enhanced also the level of the phosphorylated, active ERK kinase (Fig. 6A). To better define the signaling pathways involved in p17-Lyrm-induced B-cell clonogenicity, we cloned Raji cells in the presence of optimal concentration of the Akt inhibitor VIII. As shown in Fig 6B, the B-cell clonogenic activity of p17Lyrm was significantly inhibited by Akt inhibitor VIII, whereas the ERK1/2 inhibitor PD98059 had no effect. These findings attest for a link between clonogenicity of p17-Lyrm and activation of the Akt pathway. 


\section{Discussion}

The increased risk of lymphoma development that characterizes HIV-infected individuals is probably multifactorial and not restricted to the immune suppression caused by HIV. In particular, this notion is supported by the observation that the risk to develop distinct lymphoma histotypes is still significantly higher in the $\mathrm{HIV}^{+}$population, despite the virologic suppression and immune recovery induced by cART. ${ }^{\mathbf{1 , 2}}$ Therefore, a deeper understanding of critical pathogenic mechanisms, other than HIV-related immune suppression, which promote lymphomagenesis in the HIV setting is warranted.

We herein report on the detection and functional characterization of the dominant HIV p17 protein variant detected in the blood and tumor microenvironment of a rare case of HIV-related SMZL. Despite in this patient the levels of HIV RNA were always below the limit of detection since the beginning of cART, HIV DNA was detected in peripheral mononuclear cells, and in total and B-cell-enriched spleen cells. ${ }^{14}$ These findings were further expanded in the present study in which immunohistochemical analysis localized immunoreactivity for HIV p17 mainly in reactive follicular germinal centers. These results are consistent with previous findings indicating that HIV p17 may accumulate within lymphoid tissues, even in patients showing an apparently successful control of HIV replication induced by cART. $^{\mathbf{1 1 - 1 3}}$ Of note, persistence of p17 protein within follicular germinal centers may contribute to a local chronic stimulation of B lymphocytes, which may enhance the risk to develop a lymphoma, particularly of the marginal zone subtype. ${ }^{\mathbf{1 9 , 2 0}}$ As observed in HIV-related high-grade NHLs, ${ }^{\mathbf{3}}$ an identical dominant variant p17 sequence was found in both plasma and splenic lymphoma tissues of this patient. The presence of the same Ala-Ala 
insertion between amino acids 117 and 118 detected in two HIV-related NHLs ${ }^{9}$ prompted us to analyze in further detail the biologic properties of this p17 variant in the light of its possible contribution to the development of this SMZL.

The analysis of the possible direct B-cell growth-promoting ability of p17-Lyrm disclosed modest, although detectable increases in the proliferation of normal B lymphocytes with some variability among donors. More evident growth-promoting effects were induced by p17-Lyrm in EBV-activated and transformed B cells, suggesting that this p17 variant probably contributes more efficiently to the proliferation of B cells already committed to growth by other strong activating signals. Considering the pathogenic role of chronic antigenic stimulation in the development of marginal zone lymphomas, also including the splenic subtype, the possible role of an abnormal reactivity to an undefined antigen in this SMZL could have provided the pathogenic background favorable for a contributory role of p17-Lyrm. In keeping with this possibility, p17-Lyrm was also shown to induce a significant increase in the number of Raji cell colonies in soft agar compared with refp17, an effect similar to that induced by the vp17 NHL-a101 derived from a high-grade HIV-related NHL carrying the same Ala-Ala insertion. ${ }^{9}$

Consistent with previous findings indicating a pro-angiogenic role of HIV p17, ${ }^{\mathbf{1 2}}$ we showed that p17-Lyrm was able to promote the formation of capillary-like structures and enhance endothelial cell migration. Although an abnormal angiogenesis has been described in lymphomas, ${ }^{\mathbf{2 1}}$ the particular vascular organization of the spleen makes difficult to ascertain whether the proangiogenic properties of the p17-Lyrm could have had a role in the development of this SMZL. On the other hand, our findings further support the notion that distinct lymphoma-associated p17 variants may exert pro-angiogenic activities. 
More interestingly from a pathogenic point of view is the demonstration that, unlike the refp17, p17-Lyrm enhanced Akt and ERK kinase activation in B cells, critical pathways in lymphoma development. $^{\mathbf{2 2 , 2 3}}$ These findings further confirm the involvement of these signaling pathways in mediating the biologic effects exerted by p17 lymphomagenic variants in B lymphocytes. In particular, using pharmacologic inhibitors of these two signalings, we demonstrated that p17-Lyrm clonogenic activity is dependent on Akt activation, whereas ERK1/2 are not involved. In B lymphocytes, Akt may be activated by different upstream stimuli, most notably the B-cell receptor. $^{22}$ Therefore, p17-Lyrm could synergize with B-cell receptor signaling in boosting the Akt activation mediated by exogenous antigens. Long-term persistence of HIV p17 within lymphoid germinal centres together with a chronic antigenic stimulation may enhance B-cell activation/proliferation and favor the occurrence of secondary genetic alterations that may lead to lymphoma development.

Although SMZL are relatively rare in HIV-seropositive patients and no general conclusion can be drawn from the characterization of a single case, the SMZL investigated herein is particularly useful to uncover the possible direct contribution of HIV p17 variants to lymphoma development. In fact, the pathogenic relevance of p17-Lyrm in this case is supported not only by the critical biologic effects exerted on B cells by this protein, but also by the negativity of the patient for the main viral infections associated with lymphomagenesis, including EBV, CMV, HBV, and HCV. ${ }^{\mathbf{1 4}}$ The results presented herein confirm and extend our previous observations indicating that HIV p17 variants with distinct molecular signatures may accumulate in lymphoid tissues of HIV-infected individuals where they may act as a local stimulus promoting the development of lymphomas. ${ }^{\mathbf{9 , 2 4}}$ In addition to a pathogenic cooperation with EBV in the development of EBV-related lymphomas, ${ }^{\mathbf{1 8}}$ 
distinct HIV p17 variants may also synergize with EBV-unrelated antigens chronically persisting in the microenvironment, thus increasing the risk to develop EBV-unrelated lymphomas, also including marginal zone B-cell lymphomas. This constitutes an attractive hypothesis that needs to be thoroughly investigated by further studies. Confirmation of these findings on prospective cohorts of cases will at least in part explain why the risk of lymphoma is still high in $\mathrm{HIV}^{+}$patients despite the immunoreconstitution promoted by $\mathrm{cART}$ and will provide the rationale for new prevention strategies in at risk individuals.

\section{References}

1. Engels EA, Pfeiffer RM, Goedert JJ, Virgo P, McNeel TS, Scoppa SM, et al. Trends in cancer risk among people with AIDS in the United States 1980-2002. AIDS 2006; 20(12):1645-1654.

2. Shiels MS, Pfeiffer RM, Gail MH, Hall HI, Li J, Chaturvedi AK, et al. Cancer burden in the HIV-infected population in the United States. J Natl Cancer Inst 2011; 103(9):753-762.

3. Patel P, Hanson DL, Sullivan PS, Novak RM, Moorman AC, Tong TC, et al. Adult and Adolescent Spectrum of Disease Project and HIV Outpatient Study Investigators. Incidence of 
types of cancer among HIV-infected persons compared with the general population in the United States, 1992-2003. Ann Intern Med 2008; 148(10):728-736.

4. Gibson TM, Morton LM, Shiels MS, Clarke CA, Engels EA. Risk of non-Hodgkin lymphoma subtypes in HIV-infected people during the HAART era: a population-based study. AIDS 2014; 28(15):2313-2318.

5. Dolcetti R, Gloghini A, Caruso A, Carbone A. A lymphomagenic role for HIV beyond immune suppression? Blood 2016; 127(11):1403-1409.

6. Curreli S, Krishnan S, Reitz M, Lunardi-Iskandar Y, Lafferty MK, Garzino-Demo A, et al. B cell lymphoma in HIV transgenic mice. Retrovirology 2013; 10:92.

7. Carroll VA, Lafferty MK, Marchionni L, Bryant JL, Gallo RC, Garzino-Demo A. Expression of HIV-1 matrix protein p17 and association with B-cell lymphoma in HIV-1 transgenic mice. Proc Natl Acad Sci U S A 2016; 113(46):13168-13173.

8. Giagulli C, Marsico S, Magiera AK, Bruno R, Caccuri F, Barone I, et al. Opposite effects of HIV-1 p17 variants on PTEN activation and cell growth in B cells. PLoS One 2011; 6(3):e17831.

9. Dolcetti R, Giagulli C, He W, Selleri M, Caccuri F, Eyzaguirre LM, et al. Role of HIV-1 matrix protein p17 variants in lymphoma pathogenesis. Proc Natl Acad Sci U S A 2015; 112(46):1433114336.

10. Caccuri F, Iaria ML, Campilongo F, Varney K, Rossi A, Mitola S, et al. Cellular aspartyl proteases promote the unconventional secretion of biologically active HIV-1 matrix protein p17. Sci Rep 2016; 6:38027.

This article is protected by copyright. All rights reserved. 
11. Fiorentini S, Riboldi E, Facchetti F, Avolio M, Fabbri M, Tosti G, et al. HIV-1 matrix protein p17 induces human plasmacytoid dendritic cells to acquire a migratory immature cell phenotype. Proc Natl Acad Sci U S A 2008; 105(10):3867-3872.

12. Caccuri F, Giagulli C, Bugatti A, Benetti A, Alessandri G, Ribatti D, et al. HIV-1 matrix protein p17 promotes angiogenesis via chemokine receptors CXCR1 and CXCR2. Proc Natl Acad Sci U S A 2012; 109(36):14580-14585.

13. Popovic M, Tenner-Racz K, Pelser C, Stellbrink HJ, van Lunzen J, Lewis G, et al. Persistence of HIV-1 structural proteins and glycoproteins in lymph nodes of patients under highly active antiretroviral therapy. Proc Natl Acad Sci U S A 2005; 102(41):14807-14812.

14. Cagliuso M, Conti V, Trasarti S, Lombardi L, Riminucci M, Perez M, et al. Splenic marginal zone lymphoma in a HIV-1 infected patient: evidence favouring a pathogenetic role of HIV-1 itself in the lymphomagenesis. Infection 2013; 41(1):255-258.

15. De Francesco MA, Baronio M, Fiorentini S, Signorini C, Bonfanti C, Poiesi C, et al. HIV-1 matrix protein p17 increases the production of proinflammatory cytokines and counteracts IL-4 activity by binding to a cellular receptor. Proc Natl Acad Sci U S A 2002; 99(15):9972-9977.

16. Caruso A, Caselli E, Fiorentini S, Rotola A, Prandini A, Garrafa E, et al. U94 of human herpesvirus 6 inhibits in vitro angiogenesis and lymphangiogenesis. Proc Natl Acad Sci U S A 2009; 106(48):20446-20451.

17. Caccuri F, Giagulli C, Reichelt J, Martorelli D, Marsico S, Bugatti A, et al. Simian immunodeficiency virus and human immunodeficiency virus type 1 matrix proteins specify different capabilities to modulate B cell growth. J Virol 2014; 88(10):5706-5717. 
18. Martorelli D, Muraro E, Mastorci K, Dal Col J, Faè DA, Furlan C, et al. A natural HIV p17 protein variant up-regulates the LMP-1 EBV oncoprotein and promotes the growth of EBVinfected B-lymphocytes: implications for EBV-driven lymphomagenesis in the HIV setting. Int $J$ Cancer 2015; 137(6):1374-1385.

19. Arcaini L, Rossi D, Paulli M. Splenic marginal zone lymphoma: from genetics to management. Blood 2016; 127(17):2072-2081.

20. Smedby KE, Ponzoni M. The aetiology of B-cell lymphoid malignancies with a focus on chronic inflammation and infections. J Intern Med 2017; 282(5):360-370.

21. Ribatti D, Nico B, Ranieri G, Specchia G, Vacca A. The role of angiogenesis in human nonHodgkin lymphomas. Neoplasia 2013; 15(3):231-238.

22. Blachly JS, Baiocchi RA. Targeting PI3-kinase (PI3K), AKT and mTOR axis in lymphoma. $\mathrm{Br}$ J Haematol 2014; 167(1):19-32.

23. Spina V, Martuscelli L, Rossi D. Molecular deregulation of signaling in lymphoid tumors. Eur J Haematol 2015; 95(4):257-269.

24. Giagulli C, D'Ursi P, He W, Zorzan S, Caccuri F, Varney $\mathrm{K}$, et al. A single amino acid substitution confers B-cell clonogenic activity to the HIV-1 matrix protein p17. Sci Rep 2017; 7(1):6555.

This article is protected by copyright. All rights reserved. 


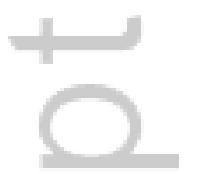

4 


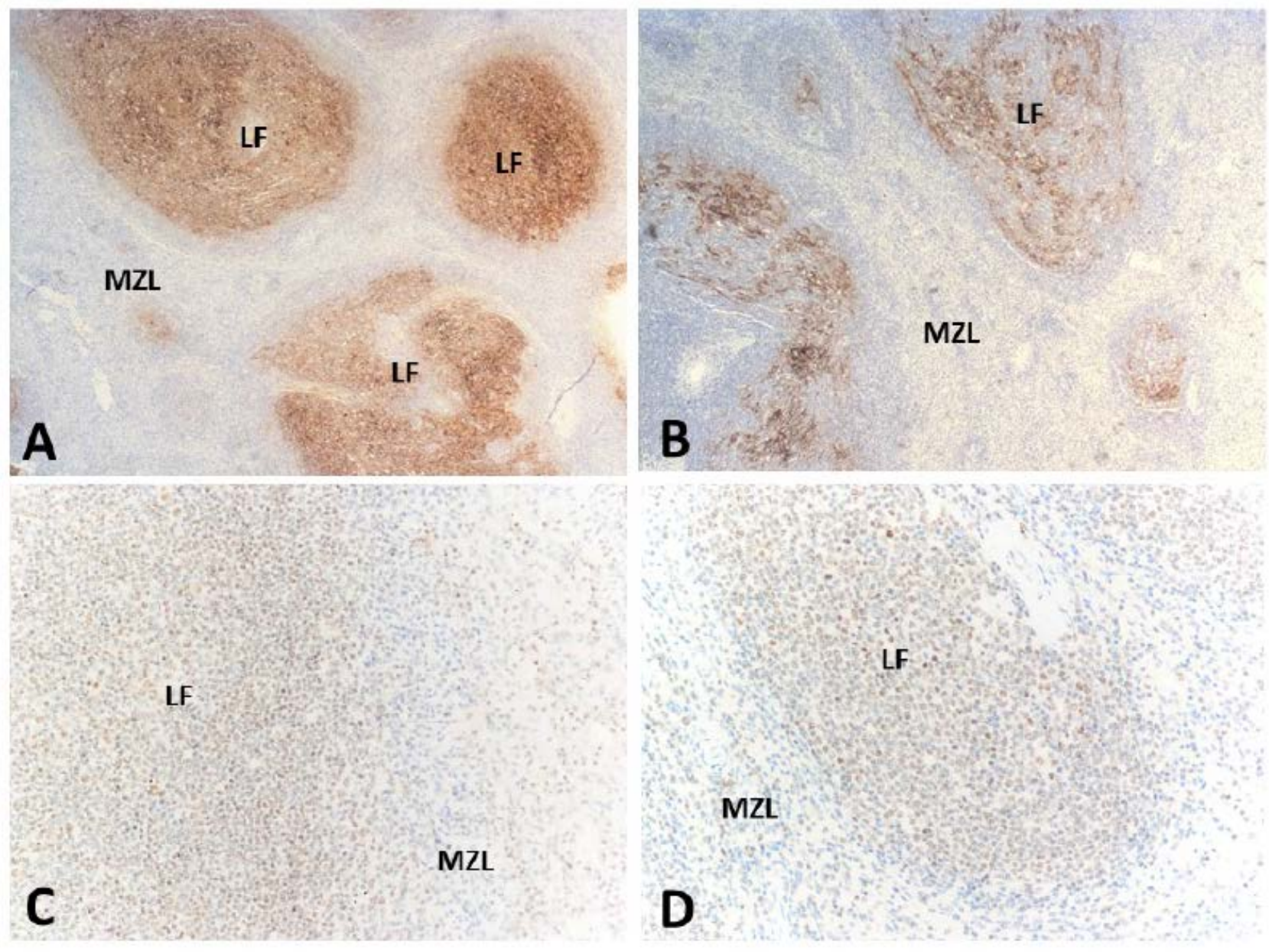

Figure 1. Detection of HIV p17 in marginal zone lymphoma of the spleen. CD21 (A) and CD23 (B) immunostain is restricted to meshworks of follicular dendritic reticulum cells (FDRC) located within splenic lymphoid follicles. Immunostaining for p17 ( $\mathbf{C}$ and $\mathbf{D})$ is present within the follicles with a pattern consistent with that of FDRCs and in normal endothelial cells located within the splenic marginal zone lymphoma. Immunostained cells show a cytoplasmic and granular pattern of staining. Lymphoma cells do not express HIV p17. Formalin fixed paraffin embedded tissue sections, Hematoxylin counterstaining. Original magnification x250. Abbreviations: LF, lymphoid follicles; MZL, marginal zone lymphoma. 
A

\begin{tabular}{|c|c|c|c|c|c|c|c|}
\hline & 10 & 20 & 30 & 40 & 50 & 60 & \\
\hline & $\ldots|\ldots|$ & $\ldots|\ldots|$ & $\ldots|\ldots|$ & $\ldots|\ldots|$ & $\ldots|\ldots|$ & $\ldots|\ldots|$ & $\ldots|\ldots|$ \\
\hline refp17 & MGARASVLSG & GELDRWEKIR & LRPGGKKKYK & LKHIVWASRE & LERFAVNPGL & LETSEGCRQI & LGQLQPSLQT \\
\hline p17- Lyrm & $\cdots \cdots \cdots$ & $\ldots \ldots \ldots$ & $\ldots \ldots \ldots R$ & . I. . . & $\ldots \ldots \ldots$ & & \\
\hline 01 & $\ldots \ldots \ldots$ & $\ldots$ K.... & $\ldots \ldots \ldots R$ & & & & \\
\hline & $\begin{array}{c}80 \\
\ldots \ldots \mid \ldots I^{80} \\
\text { GSEELRSLYN }\end{array}$ & $\begin{array}{l}0 \quad 90 \\
\ldots . .|\ldots .| \\
\text { TVATLYCVHO }\end{array}$ & $\begin{array}{c}0 \quad 100 \\
\ldots .|\ldots .| \\
\text { RIEIKDTKEA }\end{array}$ & $\begin{array}{c}110 \\
\ldots \ldots|\ldots .| \\
\text { LDKIEEEONK }\end{array}$ & $\begin{array}{l}0 \quad 117 \\
\ldots \ldots \ldots \mid \\
\text { SKKKAOO--A }\end{array}$ & $\begin{array}{c}8123128 \\
\ldots \ldots \text { I } \ldots .1 \\
\text { AADTGHSSOV }\end{array}$ & $\begin{array}{c}8132 \\
\ldots 1 \\
\text { sony }\end{array}$ \\
\hline 17- Lyrm & GSEELRSLIN & $\ldots$ v..... & K.DV...... & $\begin{array}{l}\text { LDKLEEEQNK } \\
. \mathrm{E} \ldots \ldots \ldots\end{array}$ & $\ldots$ T. . AA & AGE. . N... & SQNY \\
\hline LL-a101 & $\ldots \ldots$ к... & .I.V. . . . & $\ldots v \ldots \ldots$ & .E. . . . & C. . . AA. & $\ldots$ n. $\ldots$ & $\ldots$ \\
\hline
\end{tabular}

\section{B}

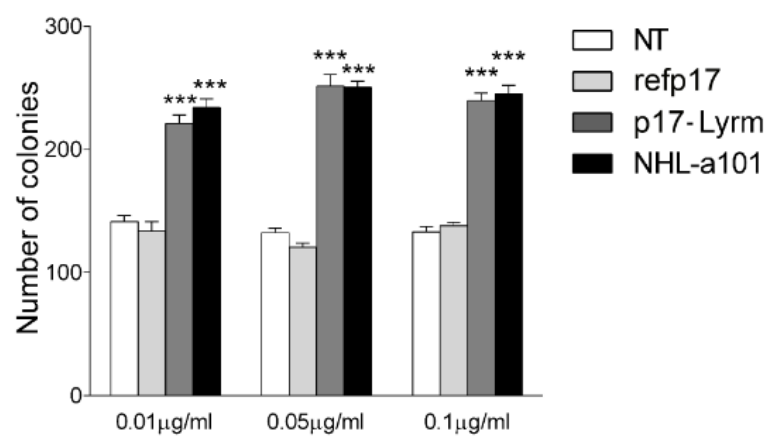

Figure 2. Alignment and comparison among amino acid sequences of refp17 and vp17s and effects of different recombinant p17s on B-cell clonogenicity. (A) Sequences are represented by the single-letter amino acid code. Amino acid positions are referred to the prototype genotype $\mathrm{B}$ strain BH10 (UniProtKB P04585; refp17), adopted as reference for this analysis. Each amino acid residue of vp17s not differing from the refp17 sequence is represented by a dot. (B) Raji cells were plated in 12-well plates, and after $4 \mathrm{~d}$, medium was replaced by fresh medium with various concentrations, $0.01,0.05,0.1 \mu \mathrm{g} / \mathrm{ml}$, of the p17 lymphoma-associated variant isolated from samples of the $\mathrm{HIV}^{+}$SMZL patient namely p17-Lyrm as indicated. Cells not treated (NT) or treated with refp17 were used as negative control, whereas cells treated with the vp17 NHL-a101 were used as positive clonogenic control. The cell growth was analyzed by using 3-[4,5-Dimethylthiazol-2y1]-2, 5-diphenyltetrazolium bromide (MTT). Data represent the average number of colonies \pm SD from three independent experiments performed in triplicate. The statistical significance between control and treated cultures was calculated using one-way ANOVA performed separately for each concentration of $\mathrm{p} 17$ variants, across the three groups. Bonferroni's post-test was used to compare data. $* * * P<0.001$. 


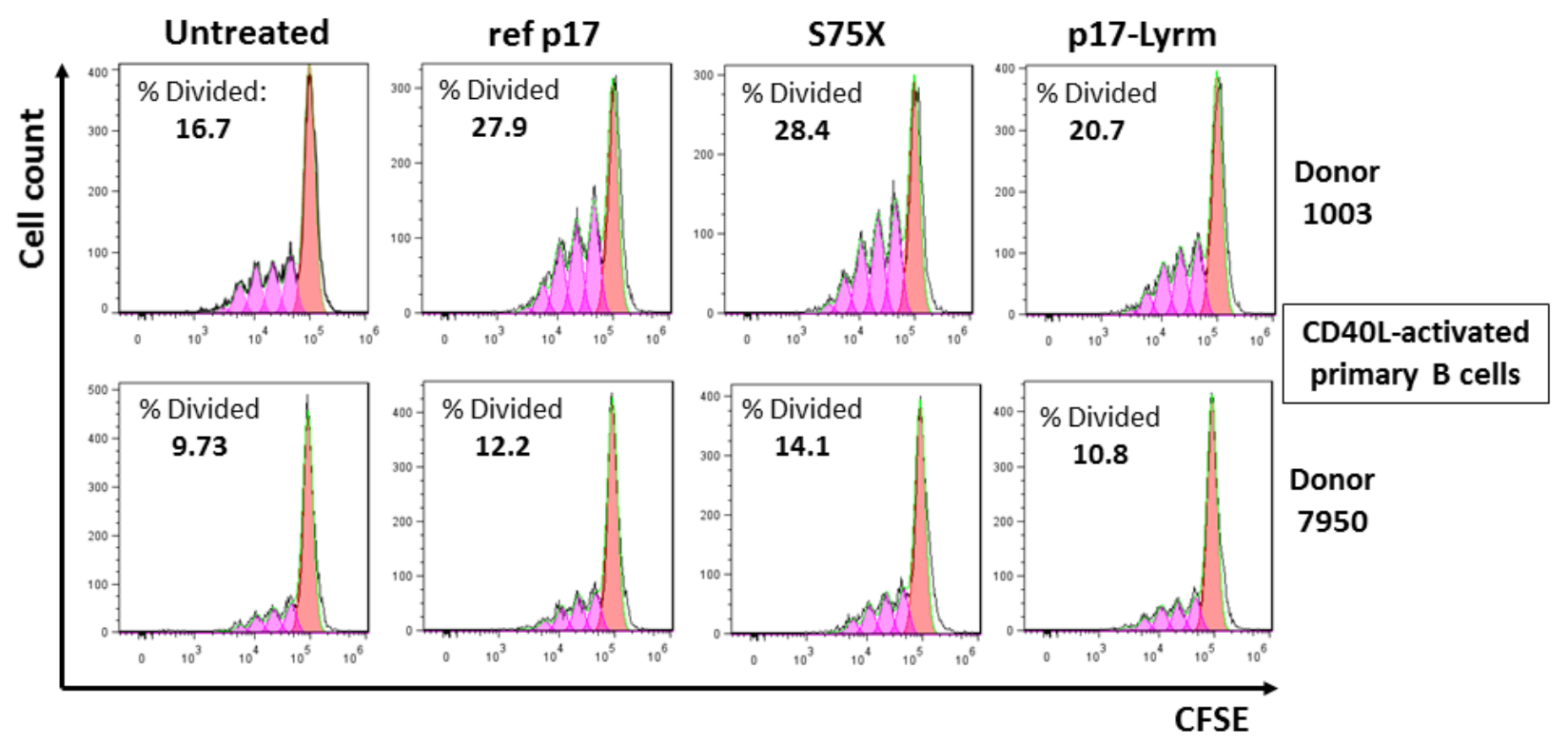

Figure 3. Effects of p17-Lyrm on the proliferation of primary $B$ cells. After activation with CD40L for 7 days, primary B cells were cultured for 3 days in the presence of ref p17, S75X or p17-Lyrm recombinant proteins $(1 \mu \mathrm{g} / \mathrm{ml})$. Exemplary proliferation analyses of carboxyfluorescein succinimidyl ester (CFSE)-labeled activated B-cells from two unrelated donors are shown. In each panel, the percentage of divided cells is indicated. 

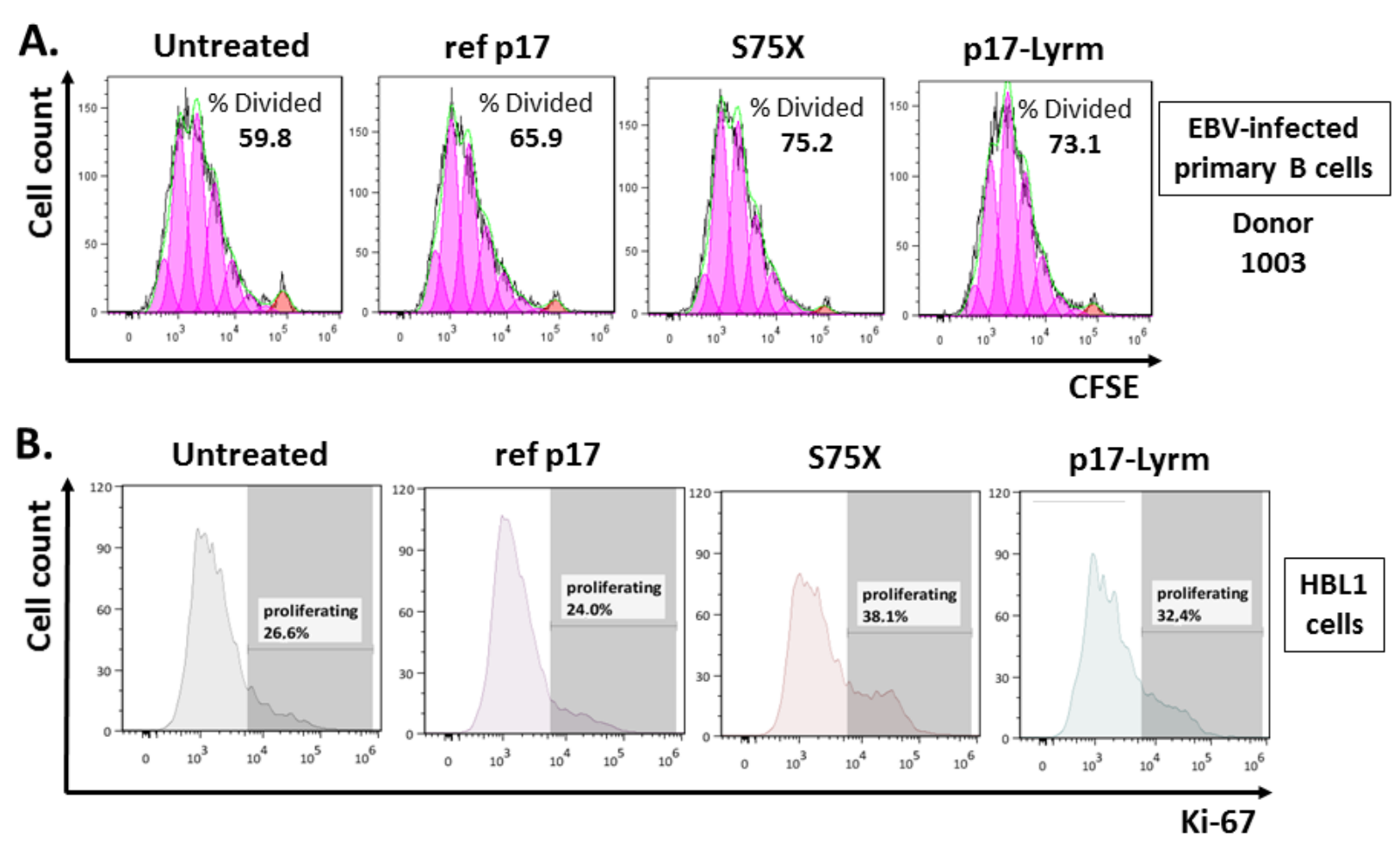

Figure 4. Effects of p17-Lyrm on the proliferation of primary EBV-infected B cells and HBL1 lymphoma cells. A. Primary B lymphocytes were immortalized with the B95.8 EBV strain for 10 days, then treated 4 days with ref $\mathrm{p} 17$, S75X or p17-Lyrm recombinant proteins $(1 \mu \mathrm{g} / \mathrm{ml})$. Exemplary proliferation analyses of carboxyfluorescein succinimidyl ester (CFSE)-labeled EBVinfected B-cells from one donor are shown. In each panel, the percentage of divided cells is indicated. B. Exemplary histogram plots of Ki-67 labeling in the HBL-1 cell line treated with ref p17, S75X or p17-Lyrm recombinant proteins $(1 \mu \mathrm{g} / \mathrm{ml})$ for 48 hours in $1 \%$ FCS. The percentage of proliferating $\mathrm{Ki}-67^{+}$cells is indicated in each panel. The results are representative of three independent experiments. 
A
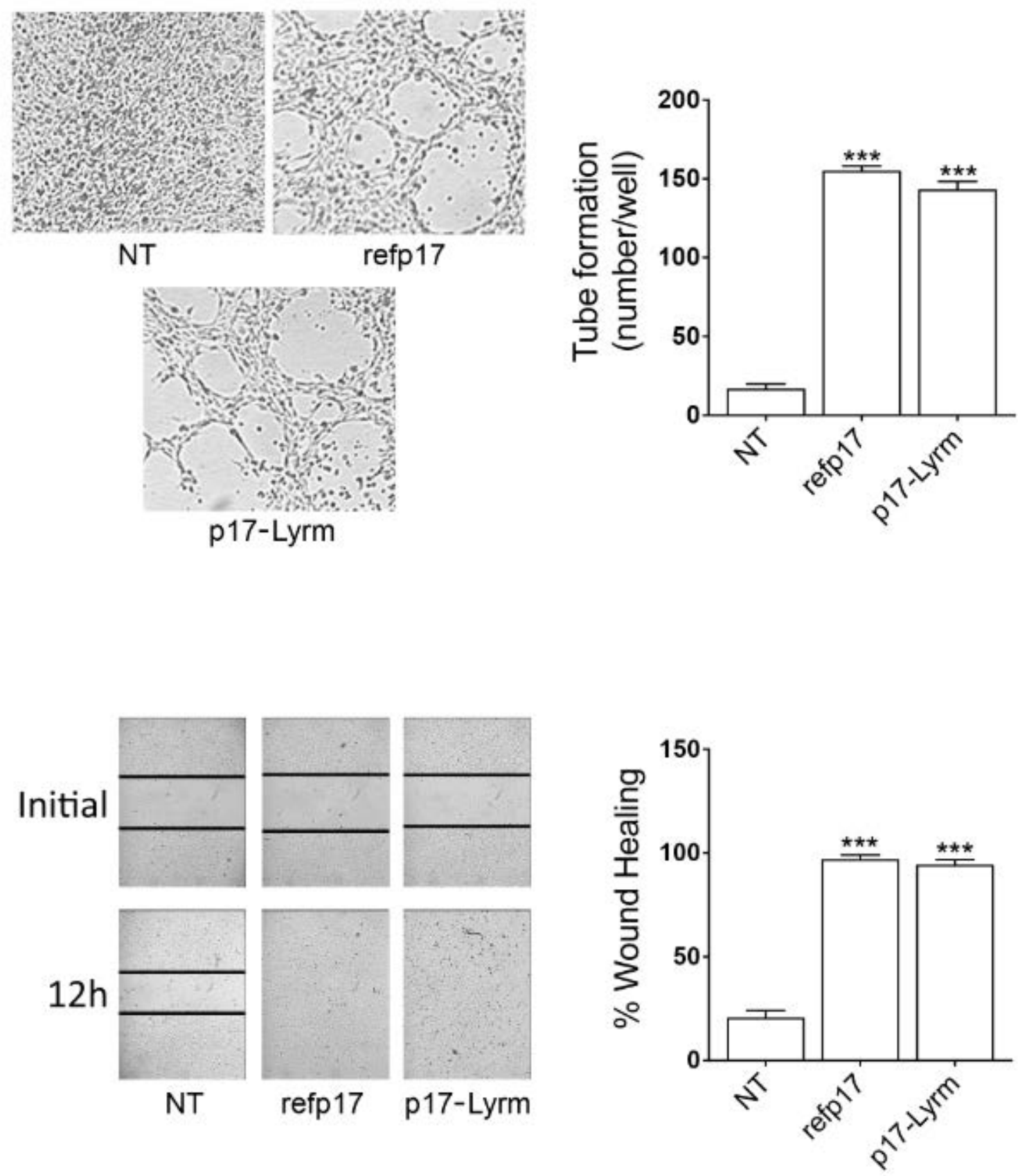

Figure 5. p17-Lyrm promotes capillary-like structures formation and cell migration.

HUVECs were stimulated for $8 \mathrm{~h}$ at $37^{\circ} \mathrm{C}$ with medium alone (not treated [NT]) or with $0.01 \mu \mathrm{g} / \mathrm{ml}$ of refp17 (as a positive control) or p17-Lyrm. Pictures are representative of 3 independent experiments with similar results (magnification $\times 10$ ). Values reported for HUVEC capillary-like structure (tubes) formation are the mean \pm SD of 3 independent experiments. Statistical analysis 
was performed by one-way ANOVA, and the Bonferroni post-test was used to compare data. *** $P$ $<0.001$. (B) Confluent HUVEC monolayers were scratched and cultured for $12 \mathrm{~h}$ at $37^{\circ} \mathrm{C}$ with medium alone (NT) or containing $0.01 \mu \mathrm{g} / \mathrm{ml}$ of refp17 (as a positive control) or p17-Lyrm. Images are representative of 3 independent experiments with similar results (magnification $\times 10$ ). Statistical analysis was performed by one-way ANOVA, and the Bonferroni post-test was used to compare data. $* * * P<0.001$. 
A

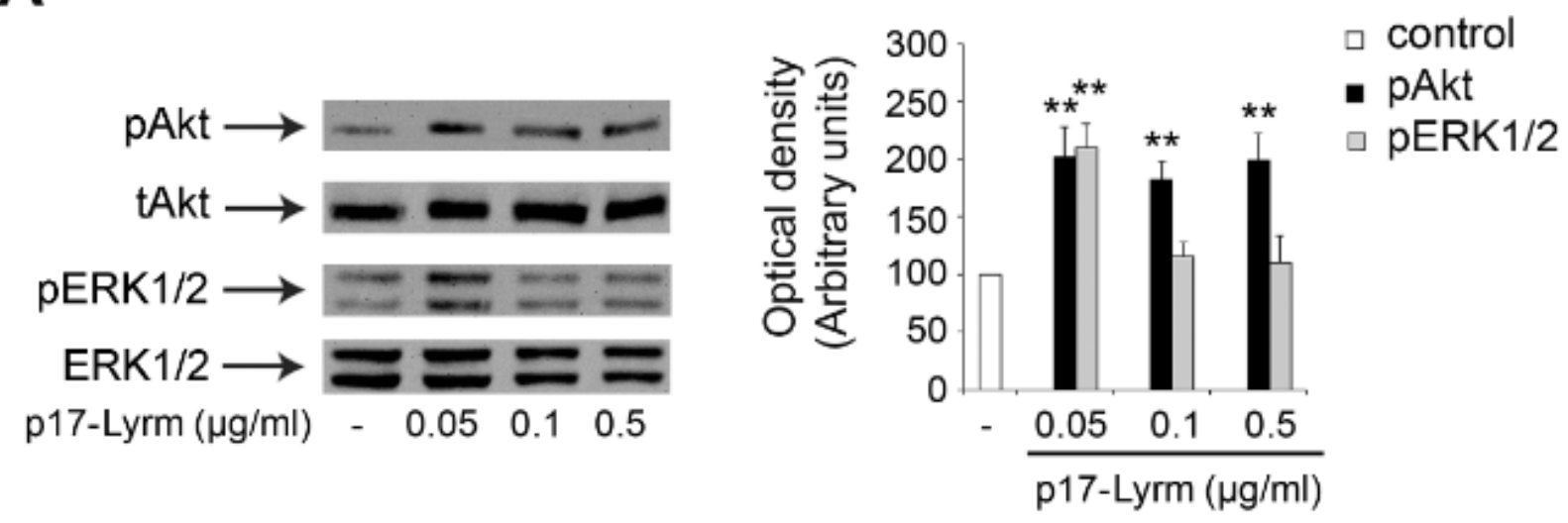

B

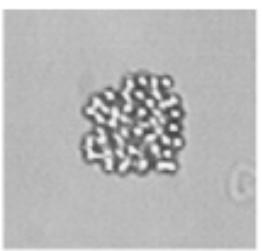

NT

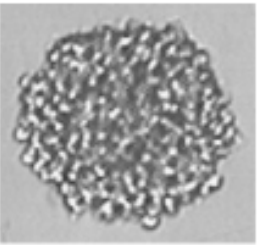

p17-Lyrm

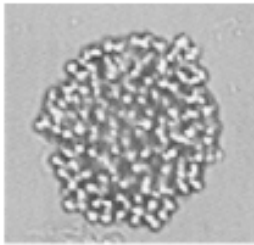

p17-Lyrm + PD98059

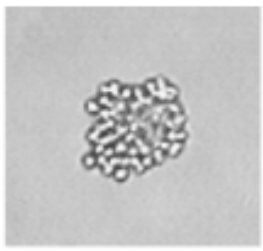

p17-Lyrm

+ Akt VIII i
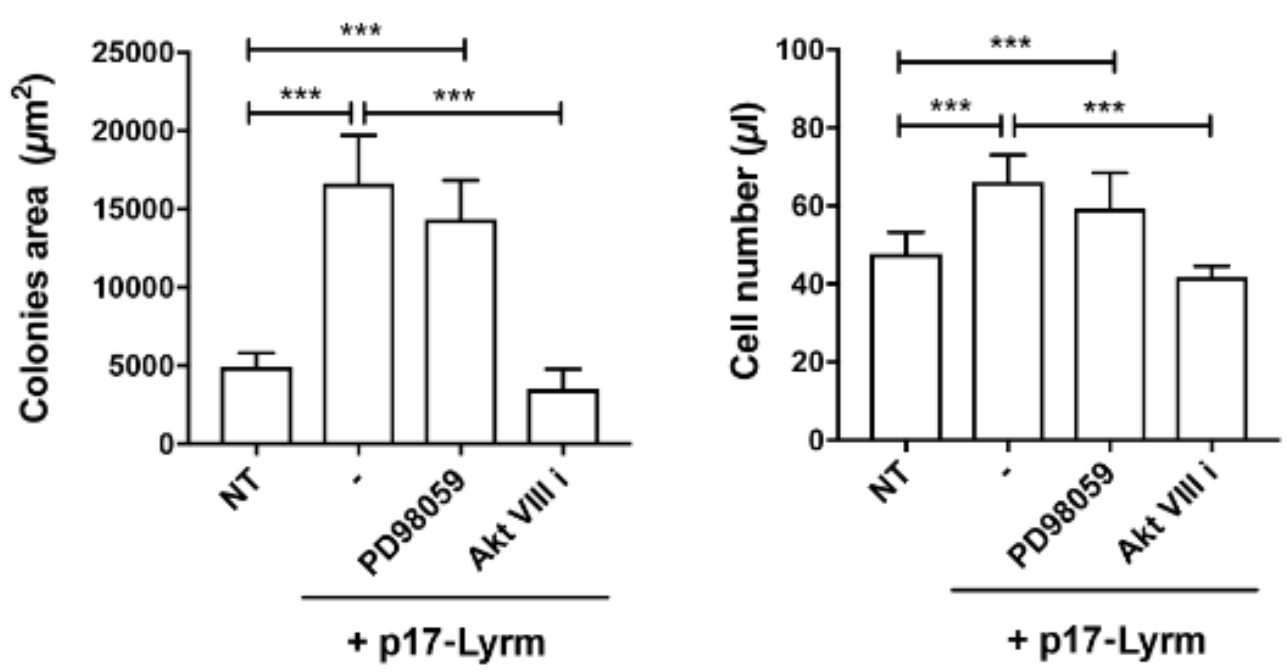

Figure 6. Effects of p17-Lyrm stimulation on Akt and ERK activity in Raji cells. (A) Raji cells were treated or not for $5 \mathrm{~min}$ with $0.05,0.1$ and $0.5 \mu \mathrm{g} / \mathrm{ml}$ of p17-Lyrm. Not treated cells were used as control (lane 1). Western blot analysis of cell lysates indicates that p17-Lyrm activates Akt and ERK1/2, as shown by the respective phosphorylation state, verified by densitometric analysis and plotting of the pAkt/totalAkt (tAkt) and pERK1/2/ERK1/2 ratios. Left panel, blots from one representative experiment of three with similar results are shown. Right panel, values reported for 
phosphorylation of Akt and ERK1/2 are the mean \pm SD of three independent experiments. Statistical analysis was performed by one-way ANOVA and the Bonferroni's post-test was used to compare data. $* * P<0.01$. (B) Raji cells were cultured for 8 days in the presence or absence of $\mathrm{p} 17$ Lyrm $(0.01 \mu \mathrm{g} / \mathrm{ml})$ alone or in combination with the inhibitor of PI3K/Akt (Akt VIII i) $(1 \mu \mathrm{M})$ or the inhibitor of (MEK)/ERK1/2 (PD98059) $(10 \mu \mathrm{M})$ signaling pathways. Bright-field images represent the characteristic morphology of 2D colonies (upper panels), one colony for each condition is shown (original magnification, x40). The colony area was measured (15 colonies/condition) by using Leica Qwin image analysis software (lower panel, left). The same number of colonies (15 colonies/condition) was aseptically harvested from 96-well plates and stained with Propidium Iodide (PI) to detect $\mathrm{PI}^{-}$viable cells by flow cytometry (lower panel, right). Absolute cell counts were obtained by the counting function of the MACSQuant ${ }^{\circledR}$ Analyzer. Cells not treated (NT) were used as negative control. Data are representative of three independent experiments performed in triplicate. The statistical significance between control and treated cultures was calculated using oneway ANOVA and the Bonferroni's post-test was used to compare data. $* * * P<0.001$. 


\section{University Library}

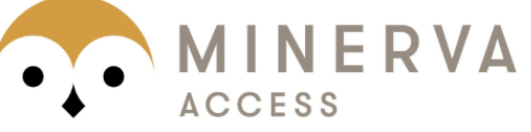

A gateway to Melbourne's research publications

Minerva Access is the Institutional Repository of The University of Melbourne

Author/s:

Caccuri, F;Muraro, E;Gloghini, A;Turriziani, O;Riminucci, M;Giagulli, C;Mastorci, K;Fae, DA;Fiorentini, S;Caruso, A;Carbone, A;Dolcetti, R

Title:

Lymphomagenic properties of a HIV p17 variant derived from a splenic marginal zone lymphoma occurred in a HIV-infected patient

Date:

2019-04-01

Citation:

Caccuri, F., Muraro, E., Gloghini, A., Turriziani, O., Riminucci, M., Giagulli, C., Mastorci, K., Fae, D. A., Fiorentini, S., Caruso, A., Carbone, A. \& Dolcetti, R. (2019). Lymphomagenic properties of a HIV p17 variant derived from a splenic marginal zone lymphoma occurred in a HIV-infected patient. HEMATOLOGICAL ONCOLOGY, 37 (2), pp.176-184. https:// doi.org/10.1002/hon.2562.

Persistent Link:

http://hdl.handle.net/11343/285140 